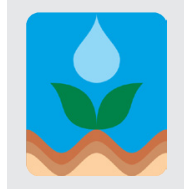

\author{
Revista Brasileira de Engenharia Agrícola e Ambiental \\ v.18, n.10, p.1072-1078, 2014 \\ Campina Grande, PB, UAEA/UFCG - http://www.agriambi.com.br \\ Protocolo 317.13 - 07/10/2013 • Aprovado em 09/05/2014
}

\title{
Climatização na pré-ordenha de vacas Girolando no inverno do semiárido
}

\author{
Luiz A. de Almeida Neto ${ }^{1}$, Héliton Pandorfi ${ }^{2}$, Gledson L. P. de Almeida ${ }^{3}$ \& Cristiane Guiselini ${ }^{4}$ \\ ${ }^{1}$ PPG em Engenharia Agrícola/UFRPE. Recife, PE. E-mail: luizz_antonio@hotmail.com \\ ${ }^{2}$ DEAGRI/UFRPE. Recife, PE. E-mail: pandorfi@deagri.ufrpe.br (Autor correspondente) \\ ${ }^{3}$ DEAGRI/UFRPE. Recife, PE. E-mail: gledson81@hotmail.com \\ ${ }^{4}$ DEAGRI/UFRPE. Recife, PE. E-mail: cguiseli@hotmail.com
}

\section{Palavras-chave:}

ambiência animal

conforto térmico

bovinos de leite

\begin{abstract}
R E S U M O
Propôs-se, neste trabalho, avaliar tempos de exposição dos animais à climatização no curral de espera sobre os índices de conforto, variáveis fisiológicas e produção de leite de vacas da raça Girolando, no inverno do semiárido pernambucano. Foram utilizadas 16 vacas com composição genética de 7/8 Holandês-Gir, divididas em quatro grupos, submetidos a três tempos de climatização 10, 20, 30 min e controle (sem climatização) adotando-se delineamento em quadrado latino 4 x 4. Para determinação da eficiência térmica da climatização foram registradas as variáveis temperatura e umidade relativa do ar, frequência respiratória, temperatura de pelame, temperatura retal e produção de leite, para os dois turnos de ordenha (manhã e tarde). A climatização por 10, 20 e 30 min mostrouse eficiente na redução da temperatura do ar no turno da tarde com redução da ordem de $1,4,2,3$ e 2,7 ${ }^{\circ} \mathrm{C}$ quando comparados com o controle, respectivamente. A climatização no curral de espera promoveu a manutenção do conforto térmico de bovinos leiteiros no inverno do semiárido pernambucano, embora sem efeito contundente nas respostas fisiológicas e de produção de leite, que não apresentaram alteração com o emprego do resfriamento adiabático evaporativo.
\end{abstract}

Key words: animal environment thermal comfort dairy cattle

\section{Pre-milking acclimatization of 'Girolando' cows during the winter in the semiarid region}

\begin{abstract}
A B S T R A C T
The objective of this study was to evaluate exposure periods of animals to acclimatization in corral on comfort indices, physiological variables and milk production of 'Girolando' cows, during the winter in the semiarid of Pernambuco. Sixteen 'Girolando' cows were used with genetic composition of 7/8 Holstein-Gir, divided in four groups, subjected to three acclimatization periods of 10, 20, $30 \mathrm{~min}$ and control (no acclimatization), adopting a $4 \times 4$ Latin square design. In order to determine acclimatization thermal efficiency, the variables temperature and relative humidity of air, respiratory frequency, haircoat temperature, retal temperature and milk production, for both milking periods (morning and afternoon) were observed. Acclimatization for 10, 20 and 30 min were efficient in dropping air temperature during the afternoon, with a decrease around $1.4,2.3$ and $2.7{ }^{\circ} \mathrm{C}$, when compared to control, respectively. Corral acclimatization promoted thermal comfort maintenance of dairy cattle in the winter in the semiarid region of Pernambuco, though there was no definite effect on physiological, behavior, production and milk composition responses, which were not altered under the adiabatic evaporative cooling.
\end{abstract}

\section{INTRODUÇÃO}

A adequação do microclima e o condicionamento térmico ambiental constituem um dos maiores desafios dos produtores de bovinos de leite no semiárido brasileiro com ênfase na minimização dos efeitos do estresse térmico animal. As condições climáticas nessas regiões podem dificultar a homeotermia com consequentes prejuízos à manutenção do equilíbrio térmico, na reprodução e na produção de leite (Perissinotto et al., 2006).
O efeito das condições desfavoráveis do ambiente pode ser atenuado pelo cruzamento de animais da espécie zebuína, mais adaptadas ao clima quente porém menos produtiva, com taurina, mais sensível a altas temperaturas; no entanto, mais produtivas. Além disto, o emprego de tecnologias como os sistemas de climatização, constitui mais uma alternativa de controle ao estresse térmico de bovinos de leite (Silva et al., 2009). Portanto, as raças bovinas necessitam de instalações e de manejo que amenizem os efeitos estressantes do ambiente. É importante dar maior atenção aos locais com grande 
concentração de animais, como a sala de espera na ordenha (Delfino et al., 2012).

A capacidade de tolerância dos animais ao estresse térmico tem sido avaliada por meio da observação de alguns parâmetros fisiológicos, como a temperatura retal e a frequência respiratória. Esses parâmetros podem ser influenciados tanto por fatores intrínsecos (idade, raça, estado fisiológico) quanto por fatores extrínsecos (hora do dia, ingestão de alimentos e de água, temperatura ambiente, velocidade do vento e estação do ano) (Perissinotto et al., 2009).

Os bovinos especializados na produção de leite possuem zona de termoneutralidade situada entre 4 e $26^{\circ} \mathrm{C}$ (Perissinotto et al., 2009) e para vacas mestiças a temperatura crítica superior da zona de termoneutralidade pode atingir valor da ordem de $29^{\circ} \mathrm{C}$, em que a homeotermia é mantida indiretamente pelos processos de transferência de calor por radiação, convecção, condução e evaporação que ocorrem na superfície do animal (Azevedo et al., 2005).

Duas estratégias fundamentais podem ser utilizadas para controlar e melhorar as condições ambientais dos animais em estresse térmico: reduzir o ganho de calor, diminuindo a carga resultante de insolação e maximizar a perda de calor pela redução da temperatura do ar e do ambiente ou promover a maior perda de energia por evaporação, diretamente pelos animais (Silva et al., 2013).

Neste contexto objetivou-se avaliar diferentes tempos de exposição dos animais à climatização no curral de espera sobre os índices de conforto, variáveis fisiológicas e produção de leite de vacas da raça Girolando, no inverno do semiárido pernambucano.

\section{Material e Métodos}

Esta pesquisa foi realizada uma propriedade comercial de produção de leite localizada no município de Capoeiras, Mesorregião Agreste e Microrregião do Vale do Ipojuca, Estado de Pernambuco, Brasil, latitude de $8^{\circ} 36^{\prime}$ 33" S, longitude de $36^{\circ} 37^{\prime} 30^{\prime \prime}$ O e altitude de $733 \mathrm{~m}$. A precipitação pluviométrica média da região é de $588 \mathrm{~mm}$ por ano com temperatura média anual de $22,1^{\circ} \mathrm{C}$. De acordo com a classificação climática de Köppen, o clima é caracterizado como Bsh, semiárido (Vianello \& Alves, 2006).

O experimento foi realizado durante a estação de inverno (julho a agosto de 2010) com duração de 56 dias divididos em 4 períodos de 14 dias utilizando-se os sete primeiros dias de cada período para adaptação dos animais aos sistemas de climatização. No decorrer dos sete dias restantes de cada período foram registradas as variáveis meteorológicas no curral de espera submetidos aos seus respectivos tempos de climatização e no ambiente externo.

Para determinação do efeito da climatização e do tempo de espera dos animais na pré-ordenha foram avaliados três tempos de climatização 10, 20 e 30 min em que os animais foram expostos ao sistema de resfriamento adiabático evaporativo (SRAE) e comparados com o controle (sem climatização) cujos animais passaram por um período de descanso de $20 \mathrm{~min}$, antes de iniciar a ordenha.

Foram utilizadas 16 vacas Girolando multíparas em lactação (50-70 dias de lactação) com composição genética de 7/8 Holandês-Gir, peso médio de $500 \mathrm{~kg}$ e produção média de leite de $18 \mathrm{~kg} \mathrm{~d}^{-1}$, sendo então divididas em quatro grupos com quatro animais para cada grupo, marcados com cabresto de cores diferentes, distribuídos por ordem de parto e estádio de lactação, o que garantiu maior homogeneidade entre os grupos.

As vacas receberam a mesma dieta alimentar em comedouro coletivo, de acordo com o manejo empregado pela fazenda. As dietas de volumosos à base de palma forrageira e capim elefante foram fornecidas em comedouro coletivo, duas vezes ao dia, sendo a primeira dieta fornecida após a primeira ordenha e a outra após a segunda ordenha. Durante o intervalo das ordenhas os animais permaneceram em área sombreada de bosque, com livre acesso à água.

Após os turnos de ordenha (manhã e tarde) os animais permaneceram em um curral de pós-ordenha (Setor 1) até que todos fossem ordenhados para, em seguida, serem liberados para a área sombreada de bosque (Setor 2) e posteriormente para a área de comedouros (Setor 3) onde recebiam as dietas de volumosos, em comedouro coletivo (Figura 1).

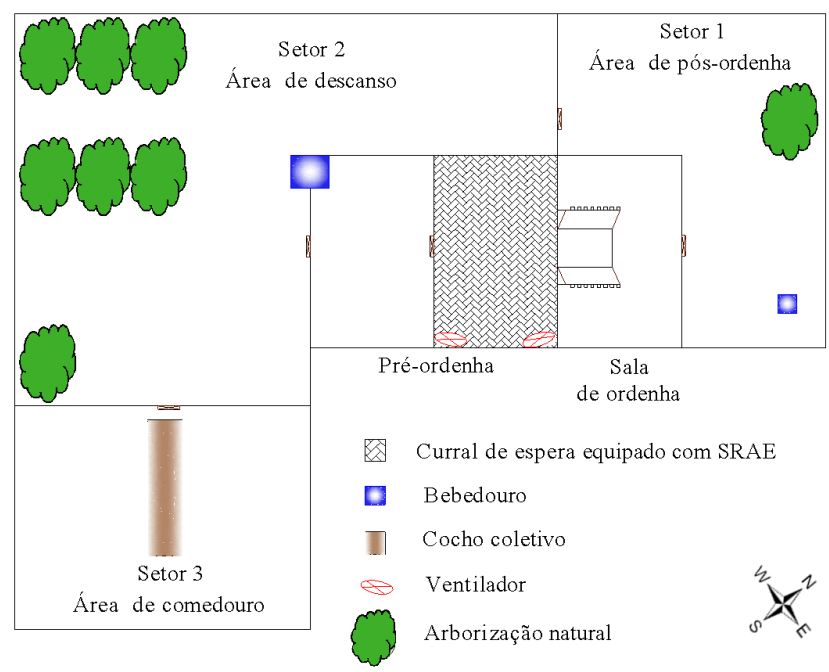

Figura 1. Esquema de localização dos setores de pósordenha, área de descanso e comedouro

O concentrado era fornecido duas vezes ao dia, na proporção de $1 \mathrm{~kg}$ de ração para cada $3 \mathrm{~kg}$ de leite produzido por vaca, em comedouros individuais, durante as ordenhas; a ração era composta basicamente de farelo de soja, farelo de algodão, farelo de milho, farelo de trigo e minerais.

O curral de pré-ordenha apresentava dimensões de $3,0 \mathrm{~m}$ de pé-direito, largura de 8 e $6 \mathrm{~m}$ de comprimento $\left(48 \mathrm{~m}^{2}\right)$ com piso de pedra rejuntada com pasta de cimento. A cobertura do curral de espera foi feita com malha preta de sombreamento (80\%) fixada em camada única sobre estrutura de madeira, sem fechamento lateral.

A ordenha era mecânica de circuito fechado realizada nos horários das 5 e $14 \mathrm{~h}$, em sistema tipo espinha de peixe com fosso central. 
O SRAE foi composto por dois ventiladores axiais da marca Ventiave ${ }^{\circ}$, modelo P3D-Plus, equipados com motor trifásico de 0,5 HP, com diâmetro de 1,0 m, vazão de $240 \mathrm{~m}^{3} \mathrm{~min}^{-1}, 965 \mathrm{RPM}$ e velocidade de deslocamento da massa de ar de até $2,5 \mathrm{~m} \mathrm{~s}^{-1}$.

Os ventiladores foram fixados na face Sul, com espaçamento de $6 \mathrm{~m}$ entre os equipamentos, a uma altura de 2,5 $\mathrm{m}$ do piso (medido a partir do centro do equipamento) e com inclinação, em relação à vertical, de $20^{\circ}$, direcionados para o piso. $\mathrm{O}$ sistema de nebulização contou com cinco linhas (tubo de polietileno), com quatro bicos nebulizadores, marca Asbrasil, modelo Hadar 7110 em cada linha, com espaçamento de 1,5 $\mathrm{m}$ entre os bicos e entre as linhas, na altura de 3,0 $\mathrm{m}$ do piso. Este sistema foi equipado com uma bomba centrífuga da marca Schneider ${ }^{\circ}$ modelo BC-92SK, de 0,75 CV e motor trifásico cujo consumo de energia era equivalente a $0,65 \mathrm{KWh}$. A vazão de água nas linhas de nebulização foi de $240 \mathrm{~L} \mathrm{~h}^{-1}$. O SRAE foi acionado manualmente e permaneceu ligado ininterruptamente, durante a permanência das vacas no curral de espera. $\mathrm{O}$ consumo médio de água pelo SRAE foi 2,0; 4,0 e 6,0 $\mathrm{L} \mathrm{vaca}^{-1}$ ordenha $^{-1}$, respectivamente, para 10, 20 e 30 min de exposição à climatização.

As variáveis meteorológicas foram registradas na préordenha e no ambiente externo, por meio de dataloggers modelo HOBO U12-12, para o registro da temperatura do ar $\left(\mathrm{Ta},{ }^{\circ} \mathrm{C}\right)$, da umidade relativa do ar (UR, \%) e da temperatura de globo negro (Tgn, ${ }^{\circ} \mathrm{C}$ ). Os sensores foram posicionados no centro geométrico da instalação, a 2,5 m do piso, no ambiente externo enquanto os sensores foram instalados no interior de um abrigo meteorológico a 1,50 $\mathrm{m}$ de altura do solo.

Para determinação da eficiência térmica proporcionado pelo SRAE foram determinados o índice de temperatura e a umidade (ITU) proposta por Thom (1959) Eq. 1 e entalpia específica (h, $\mathrm{KJ} \mathrm{kg}^{-1}$ ) proposta por Rodrigues et al. (2011) Eq. 2.

$$
\mathrm{ITU}=\mathrm{Ta}+0,36 \cdot \mathrm{Tpo}+41,5
$$

em que:

$\mathrm{Ta}$ - temperatura do ar, ${ }^{\circ} \mathrm{C}$

Tpo - temperatura de ponto de orvalho, ${ }^{\circ} \mathrm{C}$

$$
\mathrm{h}=\left\{1,006 \cdot \mathrm{Ta}+\left[\left(\frac{\mathrm{UR}}{\mathrm{Patm}}\right) \cdot 10^{\left(\frac{7,5 \cdot \mathrm{Ta}}{237,3+\mathrm{Ta}}\right)}\right]\right\} \cdot(71,28+0,052 \cdot \mathrm{Ta})
$$

em que:

Ta - temperatura do ar, ${ }^{\circ} \mathrm{C}$

Patm - pressão atmosférica do local, $\mathrm{mmHg}$

Para avaliação das variáveis fisiológicas foram registrados os dados de temperatura retal $\left(\mathrm{TR},{ }^{\circ} \mathrm{C}\right)$, frequência respiratória $\left(\mathrm{FR} ; \mathrm{mov} \mathrm{min}^{-1}\right.$ ) e temperatura de pelame $\left(\mathrm{TP},{ }^{\circ} \mathrm{C}\right)$ realizados nos horários das 5 e $14 \mathrm{~h}$, duas vezes por semana. Todos os animais foram submetidos a essas determinações, antes e depois da climatização, sendo previamente identificados de maneira que permanecessem fixos durante o período de acompanhamento.
A verificação da FR se deu a partir da contagem do número de movimentos da região do flanco realizados pelo animal, no intervalo de $1 \mathrm{~min}$. Após o registro da FR foram tomadas as medidas da TR, com auxílio de termômetro digital de uso veterinário, escala entre 20 e $50{ }^{\circ} \mathrm{C}$, introduzido no reto dos animais, durante o tempo de $1 \mathrm{~min}$ para estabilização e obtenção do valor da temperatura.

$O$ registro da TP foi realizado mediante o uso de um termômetro de infravermelho, marca ETI Ltd ${ }^{\circ}$, modelo RayTemp $^{\mathrm{TM}} 3$.

Determinou-se a temperatura média do pelame de acordo com Pinheiro et al. (2005), com registros de temperatura da cabeça, dorso, canela e úbere de cada animal, em seus respectivos tratamentos, Eq. 3.

$$
\mathrm{TP}=0,1 \cdot \mathrm{T}_{\text {cabeça }}+0,7 \cdot \mathrm{T}_{\text {dorso }}+0,12 \cdot \mathrm{T}_{\text {canela }}+0,08 \cdot \mathrm{T}_{\text {úbere }}
$$

A produção de leite (PL, kg) foi quantificada individualmente para cada animal em seu respectivo tempo de climatização, para as duas ordenhas diárias (manhã e tarde).

O delineamento experimental adotado foi o quadrado latino $4 \times 4$, utilizando-se 16 animais distribuídos aleatoriamente em 4 grupos, com 4 períodos experimentais e 4 tratamentos (10, 20 e 30 min de climatização e controle) de forma que cada grupo foi submetido a todos os tratamentos avaliados.

Para efeito de análise estatística das variáveis meteorológicas, índices de conforto, variáveis fisiológicas e produção, foram considerados os dados registrados nos quatro períodos totalizando 28 dias de registro efetivo de dados.

A análise estatística foi realizada por meio do programa computacional Statistical Analysis System (SAS, 2007) e as inferências obtidas foram avaliadas pelo teste de Tukey $(\mathrm{P}<$ 0,05).

As variáveis foram analisadas segundo o modelo estatístico Eq. 4:

$$
\mathrm{Y}_{\mathrm{ijk}}=\mu+\mathrm{a}_{\mathrm{i}}+\mathrm{t}_{\mathrm{j}}+\mathrm{b}_{\mathrm{k}}+\mathrm{e}_{\mathrm{ijk}}
$$

em que:

$\mathrm{Y}_{\mathrm{ijk}}$ - observação da i-ésima linha e k-ésima coluna para o j-ésimo tratamento

$\begin{array}{ll}\mu & \text { - média geral } \\ a_{i} & \text { - efeito da i-ésima linha } \\ t_{j} & \text { - efeito do j-ésimo tratamento } \\ b_{k} & \text { - efeito da k-ésima coluna } \\ e_{i j k} & \text { - erro aleatório. }\end{array}$

\section{Resultados e Discussão}

Observa-se, na Tabela 1, que os valores médios para temperatura do ar ( $\mathrm{Ta}$ ) registrados no curral de espera para os diferentes tempos de climatização e controle no turno da manhã, não apresentaram diferença significativa $(\mathrm{P}>0,05)$; portanto, todas as condições de alojamento dos animais se mantiveram dentro da zona de conforto térmico (ZCT) entre 4 e $26^{\circ} \mathrm{C}$ (Perissinotto et al., 2009) independente do emprego 
Tabela 1. Valores médios e desvio padrão das variáveis ambientais e dos índices de conforto térmico registrados no interior do curral de espera nos turnos da manhã e da tarde

\begin{tabular}{|c|c|c|c|c|c|}
\hline \multirow{2}{*}{ Variáveis } & \multicolumn{4}{|c|}{ Tratamentos } & \multirow{2}{*}{$\begin{array}{l}\text { CV } \\
\text { (\%) }\end{array}$} \\
\hline & Controle & $10 \mathrm{~min}$ & $20 \mathrm{~min}$ & $30 \mathrm{~min}$ & \\
\hline \multicolumn{6}{|c|}{ Turno da manhã } \\
\hline $\mathrm{Ta}\left({ }^{\circ} \mathrm{C}\right)$ & $19,5 a \pm 0,69$ & $18,4 \mathrm{a} \pm 1,52$ & $17,9 a \pm 1,44$ & $18,2 \mathrm{a} \pm 0,52$ & 6,52 \\
\hline UR $(\%)$ & $87,5 a \pm 0,70$ & $86,0 a \pm 6,58$ & $88,5 a \pm 6,78$ & $93,2 \mathrm{a} \pm 0,35$ & 5,66 \\
\hline ITU & $67,0 a \pm 0,91$ & $66,0 a \pm 1,62$ & $65,8 \mathrm{a} \pm 1,52$ & $65,8 \mathrm{a} \pm 0,68$ & 1,98 \\
\hline $\mathrm{h}\left(\mathrm{KJ} \mathrm{kg}^{-1}\right)$ & $54,4 a \pm 2,05$ & $50,6 a \pm 6,61$ & $49,7 \mathrm{a} \pm 6,42$ & $52,3 a \pm 1,55$ & 9,01 \\
\hline \multicolumn{6}{|c|}{ Turno da tarde } \\
\hline $\mathrm{Ta}\left({ }^{\circ} \mathrm{C}\right)$ & $25,9 a \pm 0,57$ & $24,5 b \pm 0,71$ & $23,6 c \pm 0,58$ & $23,2 d \pm 0,56$ & 5,01 \\
\hline UR (\%) & $67,5 c \pm 2,49$ & $74,3 b \pm 1,90$ & $76,0 a b \pm 5,48$ & $81,0 a \pm 1,83$ & 7,87 \\
\hline ITU & $74,5 a \pm 0,67$ & $73,0 b \pm 0,91$ & $72,3 b c \pm 0,79$ & $72,0 \mathrm{c} \pm 0,79$ & 1,73 \\
\hline $\mathrm{h}\left(\mathrm{KJ} \mathrm{kg}^{-1}\right)$ & $65,9 a \pm 1,72$ & $64,8 a b \pm 2,37$ & $62,7 b \pm 2,52$ & $64,0 a b \pm 2,32$ & 3,65 \\
\hline
\end{tabular}

Médias seguidas das mesmas letras nas mesmas linhas não diferem entre si no nível de 0,05 de probabilidade, pelo teste de Tukey.

da climatização. No turno da tarde o sistema de climatização foi eficiente na redução da Ta, que apresentou efeito significativo $(\mathrm{P}<0,05)$ entre os tempos de climatização de 10, 20 e $30 \mathrm{~min}$, com valores abaixo de $26^{\circ} \mathrm{C}$. Os tempos de exposição ao sistema de resfriamento adiabático evaporativo (SRAE) por 10, 20 e 30 min se mostraram eficientes na redução dessa variável com decréscimo da ordem de $1,4,2,3$ e $2,7^{\circ} \mathrm{C}$, quando comparados com o controle, respectivamente.

A Ta observada no curral de espera climatizado corrobora com estudos realizados por Almeida et al. (2011) e Silva et al. (2011) que verificaram redução na Ta de 4,9 e $4,6^{\circ} \mathrm{C}$ no curral de espera equipado com SRAE, quando comparado com o ambiente sem climatização. A magnitude de redução na Ta está relacionada com a eficiência do SRAE em que, no presente estudo, a demanda atmosférica para evaporação foi comprometida pela condição climática apresentada na estação de inverno, com características determinadas por temperatura amenas e umidade relativa do ar elevada.

Os valores de umidade relativa do ar (UR, \%) registrados no turno da manhã (Tabela 1) não mostraram diferenças $(\mathrm{P}>0,05)$; entretanto, todas as condições de alojamento apresentaram valores acima de $70 \%$, considerados valor limite de conforto com potencial de redução do índice de temperatura e umidade (ITU) inferior a 10\% (Almeida et al., 2011). No turno da tarde a UR registrada nas condições de climatização aumentou significativamente $(\mathrm{P}<0,05)$ em decorrência do tempo de funcionamento do SRAE, quando comparados com o controle. Nota-se que os tempos de exposição de 10, 20 e 30 min excederam o valor limite para vacas em lactação acima, portanto, da zona de conforto térmico porém para Perissinotto \& Moura (2007) quando a Ta se encontra próximo ao limite superior da zona de termoneutralidade $\left(26^{\circ} \mathrm{C}\right)$ independente dos valores de UR, a sensação de conforto térmico para vacas em lactação é muito boa.

Almeida et al. (2011) verificaram umidade relativa elevada com emprego do SRAE no curral de espera. Os valores encontrados foram 69,$5 ; 74,8$ e 77,5\%, respectivamente, para os tratamentos 10, 20 e 30 min de climatização, para o verão do semiárido pernambucano.

Observou-se que não houve redução significativa $(\mathrm{P}>0,05)$ entre o controle e os tempos de climatização para o índice de temperatura e umidade (ITU), no turno da manhã (Tabela 1), o ambiente apresentou condições ideais para vacas mestiças em lactação de composição genética 7/8 Holandês-Gir que, segundo Azevedo et al. (2005) baseados na temperatura retal, o valor do ITU para que os animais expressem seu máximo potencial produtivo é de 75 .

No turno da tarde a climatização do ambiente por 10, 20 e 30 min proporcionou redução significativa $(P<0,05)$ para o ITU quando comparados com os do controle (Tabela 1). A climatização por 30 min apresentou redução significativa ( $\mathrm{P}$ $<0,05$ ), quando comparada com a de $10 \mathrm{~min}$; mas todas as condições de alojamento estiveram abaixo do valor considerado crítico (ITU < 75) para vacas 7/8 Holandês-Gir (Azevedo et al., 2005). Os resultados apontados no turno da tarde estão abaixo daqueles observados por Silva et al. (2011) que verificaram, às 14 h, valores de 78,6; 74,9;74,4 e 74,1, para o curral de espera sem climatização, 20, 30 e 40 min de climatização, com acionamento automatizado.

Os valores médios expressos pela entalpia específica (h) não apontam redução significativa $(\mathrm{P}>0,05)$ no turno da manhã para 10, 20 e 30 min de climatização e o controle (Tabela 1). Todas as condições de estudo apresentaram entalpia abaixo do limite de conforto de $67,4 \mathrm{KJ} \mathrm{kg}^{-1}$ de ar seco, obtido a partir da Ta de $26^{\circ} \mathrm{C}$ e UR de $70 \%$. O sistema de climatização promoveu, no turno da tarde, redução significativa $(\mathrm{P}<0,05)$ na entalpia entre o controle e 20 min de climatização; no entanto, embora todas as condições de estudo tenham permanecido abaixo do valor crítico. As médias de entalpia observadas neste estudo para o turno da tarde estão abaixo das obtidas por Almeida et al. (2011) que apresentaram valores da ordem de 70,5; 68,7; 66,9 e 65,8 para o controle, 10, 20 e 30 min de climatização, no verão do semiárido pernambucano.

Para períodos em que as condições de temperatura e umidade forem elevadas, poderá ocorrer limitação no uso do sistema de resfriamento adiabático evaporativo uma vez que o ar saturado irá inibir a evaporação da água. Porém durante os meses mais quentes e menos úmidos este sistema apresentará, sem dúvida, melhor desempenho (Silva et al., 2011).

Nota-se, na Figura 2A, que a variação da Ta em todos os tratamentos, incluindo o ambiente externo, atendeu à exigência térmica dos animais permanecendo entre os limites de conforto térmico de 4 a $26^{\circ} \mathrm{C}$ (Perissinotto et al., 2009) demonstrando que a condição de conforto no curral de espera foi atendida, 

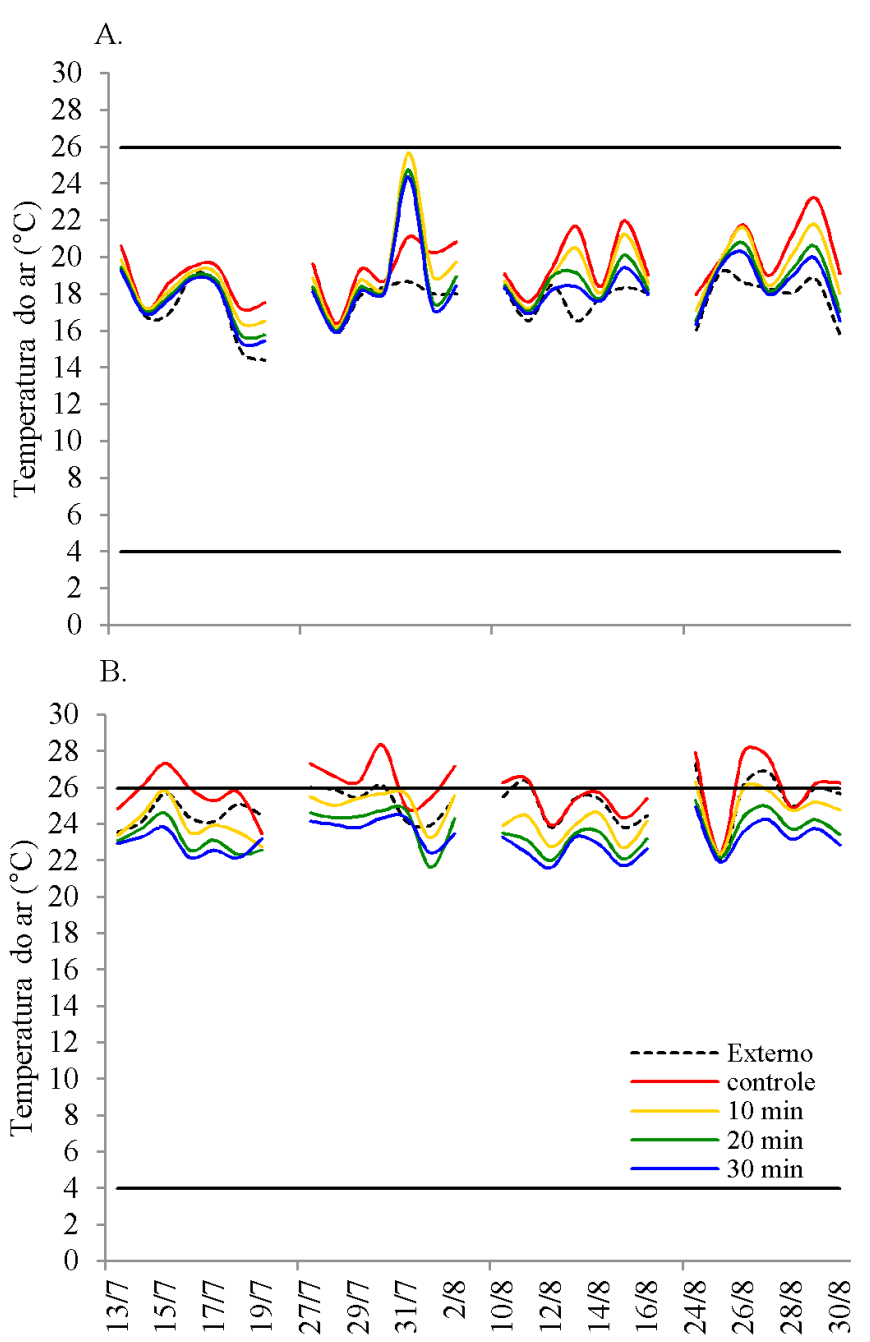

Figura 2. Variação da temperatura do ar nos diferentes tratamentos e no abrigo meteorológico (ambiente externo) no turno da manhã (A) e da tarde (B)

independente da climatização e do tempo de exposição dos animais ao SRAE. Verifica-se mesmo assim, na Figura 2B que o controle e a climatização por $10 \mathrm{~min}$ apresentaram, respectivamente, valores da ordem de 50 e 3,7\% dos dias de estudo fora da faixa de conforto recomendada enquanto nos tratamentos 20 e $30 \mathrm{~min}$ as temperaturas registradas no interior do curral de espera estiveram abaixo do limite crítico de 26 ${ }^{\circ} \mathrm{C}$; entretanto, abaixo do valor estabelecido por Azevedo et al. (2005) para vacas mestiças $\left(29^{\circ} \mathrm{C}\right)$.

Observa-se, na Figura 3A, que a variação da umidade relativa em todas as condições de estudo, incluindo o ambiente externo, se encontrava acima de 70\%, considerado valor limite para o conforto de vacas lactantes em clima quente (Matarazzo et al., 2007). No turno da tarde a UR ficou abaixo dos $70 \%$ para o ambiente com 10 min de climatização em apenas $21 \%$ dos dias de estudo; para as demais condições de climatização não ocorreram valores menores que o limite de conforto; já para o controle e para ambiente o externo, a UR não atingiu o limite inferior de 50\% (Figura 3B).

A frequência respiratória (FR) no turno da manhã (Tabela 2) apontou diferença significativa $(P<0,05)$ entre os animais
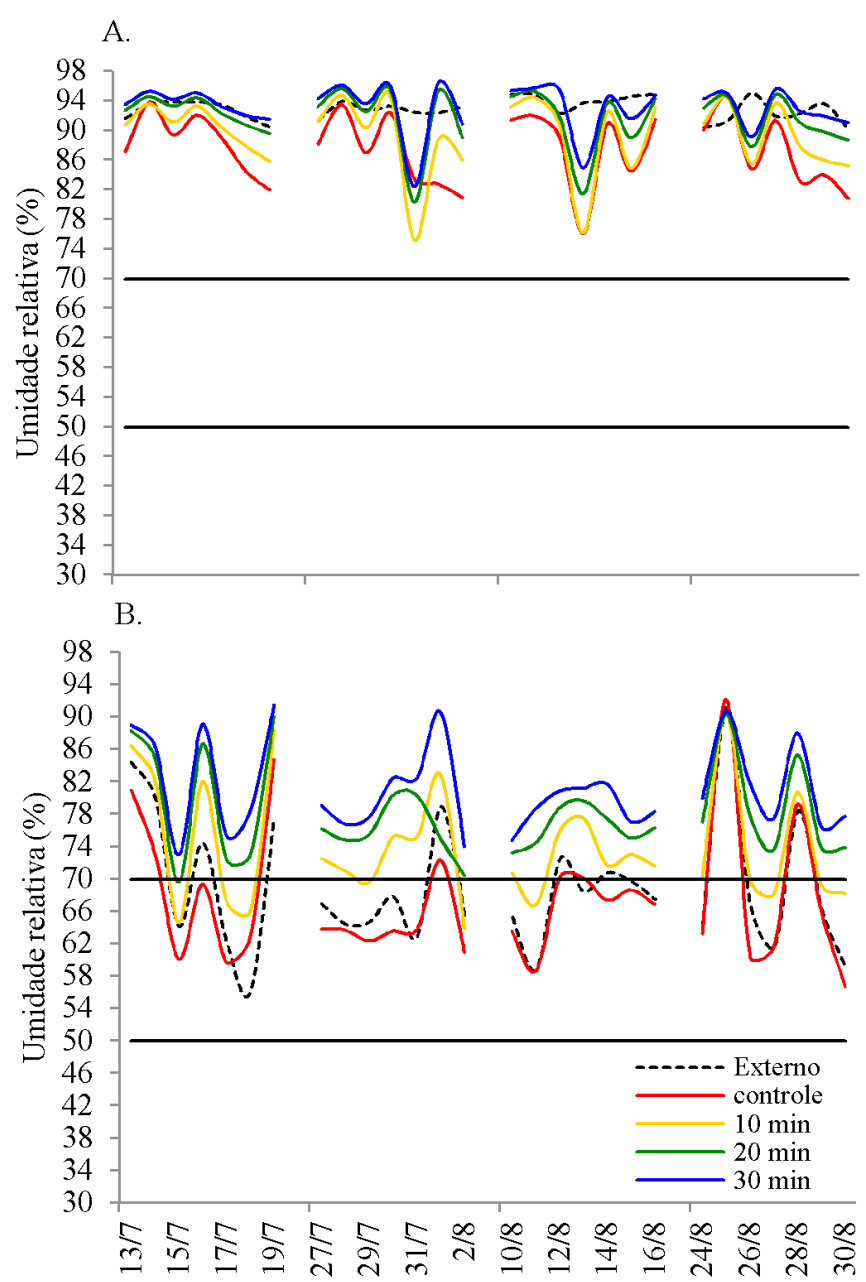

Figura 3. Variação da umidade relativa nos diferentes tratamentos e no abrigo meteorológico (ambiente externo) no turno da manhã (A) e da tarde (B)

submetidos ao controle e 30 min de climatização; apresentando valores da ordem de 28,0 e $26,0 \mathrm{mov} \mathrm{min}^{-1}$, respectivamente, tais valores de FR para vacas em lactação são considerados normais, entre 18 e 60 mov $\mathrm{min}^{-1}$, de acordo com Hahn et al. (1997) sinalizando que os animais se mantiveram livres de estresse térmico, mesmo sem climatização.

No turno da tarde também se constataram diferenças ( $P$ $<0,05)$ na FR entre os animais submetidos à climatização $(10,20$ e $30 \mathrm{~min})$ comparados ao controle apresentando valores da ordem de $38,8,33,8,31,5$ e $51,8 \mathrm{mov} \mathrm{min}^{-1}$, respectivamente (Tabela 2). Resultados semelhantes foram obtidos por Silva et al. (2011) que verificaram redução na FR de 75,7 para $34,0 \mathrm{mov} \mathrm{min}^{-1}$, para vacas lactantes da raça Girolando, que tiveram acesso ao curral de espera equipado com SRAE, durante $40 \mathrm{~min}$ de exposição, no verão do semiárido pernambucano.

Constatou-se que os valores médios da temperatura de pelame (TP) não apresentaram diferenças significativas ( $\mathrm{P}$ $>0,05)$ entre as condições de estudo, para o turno da manhã (Tabela 2); no turno da tarde as médias da TP indicaram redução significativa $(\mathrm{P}<0,05)$ entre os animais submetidos a 10,20 e 30 min de climatização, quando comparados com o controle, que apresentaram valores da ordem de 30,2, 29,5, 
Tabela 2. Valores médios e desvio padrão dos parâmetros fisiológicos avaliados nos diferentes tratamentos, nos turnos da manhã e da tarde

\begin{tabular}{|c|c|c|c|c|c|}
\hline \multirow{2}{*}{ Variáveis } & \multicolumn{4}{|c|}{ Tratamentos } & \multirow{2}{*}{$\begin{array}{l}\text { CV } \\
\text { (\%) }\end{array}$} \\
\hline & Controle & $10 \mathrm{~min}$ & $20 \mathrm{~min}$ & $30 \mathrm{~min}$ & \\
\hline & & Turno da & & & \\
\hline $\mathrm{FR}\left(\mathrm{mov} \mathrm{min}^{-1}\right)$ & $\pm 1,32 \mathrm{a}$ & $\pm 2,42 a b$ & $\pm 2,33 \mathrm{ab}$ & $\pm 2,71 \mathrm{~b}$ & 8,05 \\
\hline $\operatorname{TR}\left({ }^{\circ} \mathrm{C}\right)$ & $38,0 \pm 0,20 \mathrm{a}$ & $38,2 \pm 0,11 \mathrm{a}$ & $38,1 \pm 0,17 \mathrm{a}$ & $38,0 \pm 0,10 a$ & 0,42 \\
\hline PL (kg) & $11,691 \pm 0,73 a$ & $11,765 \pm 0,37 \mathrm{a}$ & $11,770 \pm 0,96 \mathrm{a}$ & $11,742 \pm 0,96 \mathrm{a}$ & 6,06 \\
\hline \multicolumn{6}{|c|}{ Turno da tarde } \\
\hline $\operatorname{TR}\left({ }^{\circ} \mathrm{C}\right)$ & $39,0 \pm 0,23 a$ & $38,7 \pm 0,17 \mathrm{a}$ & $38,8 \pm 0,30 \mathrm{a}$ & $38,6 \pm 0,15 \mathrm{a}$ & 0,58 \\
\hline PL (kg) & $7,666 \pm 0,61 \mathrm{a}$ & $7,609 \pm 0,36 \mathrm{a}$ & $7,610 \pm 0,35 a$ & $7,696 \pm 0,54 \mathrm{a}$ & 5,68 \\
\hline
\end{tabular}

Médias seguidas das mesmas letras nas mesmas linhas não diferem entre si no nível de 0,05 de probabilidade, pelo teste de Tukey

28,4 e $34,2^{\circ} \mathrm{C}$, respectivamente, sem efeito significativo entre os animais com climatização. Os valores médios de TP obtidos para 10, 20 e 30 min de climatização no turno da tarde estão abaixo de $32,0{ }^{\circ} \mathrm{C}$, obtidos por Silva et al. (2011), para bovinos leiteiros submetidos a climatização por $40 \mathrm{~min}$.

A temperatura retal (TR) se manteve dentro dos valores fisiológicos normais $\left(38\right.$ a $\left.39^{\circ} \mathrm{C}\right)$ no turno da manhã e tarde, conforme Perissinotto \& Moura (2007) que também não verificaram diferenças significativas $(P>0,05)$ para os valores médios nos animais do controle, 10, 20 e 30 min de climatização. A manutenção da temperatura retal evidencia que o microclima no interior do curral de espera e os mecanismos de liberação de calor foram suficientes para manter a homeotermia, independente do sistema de climatização.

Avaliando a produção de leite no turno da manhã (Tabela 2) verifica-se que não houve diferença significativa $(\mathrm{P}>0,05)$ entre os animais do controle 10,20 e $30 \mathrm{~min}$, que apresentaram valores da ordem de 11,691, 11,765, 11,770 e 11,742, respectivamente. Resultado similar também foi observado no turno da tarde, ao apresentar valores da ordem de 7,666, 7,609, 7,610 e 7,696 $\mathrm{kg} \mathrm{d}^{-1}$ para controle, 10, 20 e 30 min de climatização, respectivamente.

Os resultados apontam que os animais estavam submetidos à sua condição de conforto, independente da climatização. Esta resposta produtiva se respalda principalmente quando se compara a produção de leite verificada no verão, que apresentou valor médio para o controle de $17,53 \mathrm{~kg} \mathrm{~d}^{-1}$ (Almeida et al., 2013); portanto, nota-se incremento de $10,4 \%$ na produção de leite para a estação de inverno $\left(19,36 \mathrm{~kg} \mathrm{~d}^{-1}\right)$. A produção de leite no turno da tarde não apresentou diferença significativa $(\mathrm{P}>0,05)$ entre os tratamentos.

\section{Conclusão}

A climatização no curral de espera promoveu manutenção do conforto térmico de bovinos leiteiros no inverno do semiárido pernambucano, no turno da tarde porém sem efeito contundente nas respostas fisiológicas e de produção de leite, que não apresentaram alteração com o emprego do resfriamento adiabático evaporativo.

\section{Literatura Citada}

Almeida, G. L. P.; Pandorfi, H.; Barbosa, S. B. P.; Guiselini, C.; Almeida, G. A. P. Comportamento, produção e qualidade do leite de vacas Holandês-Gir com climatização no curral. Revista Brasileira de Engenharia Agrícola e Ambiental, v.17, p.892-899, 2013.

Almeida, G. L. P.; Pandorfi, H.; Guiselini, C.; Henrique, H. M.; Almeida, G. A. P. Uso do sistema de resfriamento adiabático evaporativo no conforto térmico de vacas da raça girolando. Revista Brasileira de Engenharia Agrícola e Ambiental, v.15, p.754-760, 2011.

Azevedo, M.; Pires, M. F. A.; Saturnino, H. M.; Lana, A. M. Q.; Sampaio, I. B. M.; Monteiro, J. B. N.; Morato, L. E. Estimativas de níveis críticos superiores do índice de temperatura e umidade para vacas leiteiras 1/2, 3/4 e 7/8 Holandês-Zebu, em lactação. Revista Brasileira de Zootecnia, v.34, p.20002008, 2005.

Delfino, L. J. B.; Souza, B. B.; Rosangela, M. N.; Silva, W. W. Influência bioclimatológica sobre os parâmetros hematológicos de bovinos leiteiros. Revista Agropecuária Científica no Semiárido, v.8, p.23-27, 2012.

Hahn, G. L.; Parkhurrst, A. M.; Gaughan, J. B. Cattle respiration rate as a function of ambient temperature. Transactions of American Society of Agricultural Engineering, v.40, p.97121, 1997.

Matarazzo, S. V.; Silva, I. J. O.; Perissinotto, M.; Fernandes, S. A. A; Arcaro Junior, I.; Arcaro, J. R. P. Eficiência de sistemas de climatização na área de descanso em instalação do tipo freestall e sua influência nas respostas produtivas e fisiológicas de vacas em lactação. Boletim de Indústria Animal, v.64, p.221-232, 2007.

Perissinotto, M.; Moura, D. J. Determinação do conforto térmico de vacas leiteiras utilizando a mineração de dados. Revista Brasileira de Engenharia de Biossistemas, v.1, p.117-126, 2007.

Perissinotto, M.; Moura, D. J.; Cruz V. F.; Souza, S. R. L.; Lima, K. A. O.; Mendes, A. S. Conforto térmico de bovinos leiteiros confinados em clima subtropical e mediterrâneo pela análise de parâmetros fisiológicos utilizando a teoria dos conjuntos fuzzy. Ciência Rural, v.39, p.1492-1498, 2009. 
Perissinotto, M.; Moura, D. J.; Matarazzo, S. V.; Silva, I. J. O.; Lima, K. A. O. Efeito da utilização de sistemas de climatização nos parâmetros fisiológicos do gado leiteiro. Engenharia Agrícola, v.26, p.663-671, 2006.

Pinheiro, M. G.; Nogueira, J. R.; Lima, M. L. P.; Leme, P. R.; Macari, M.; Näas, I. A.; Laloni, L. A.; Titto, E. A. L.; Pereira, A. F. Efeito do ambiente pré-ordenha (sala de espera) sobre a temperatura da pele, a temperatura retal e a produção de leite de bovinos da raça Jersey. Revista Portuguesa de Zootecnia, v.12, p.37-43, 2005.

Rodrigues, V. C.; Silva, I. J. O.; Vieira, F. M. C.; Nascimento, S. T. A correct enthalpy relationship as thermal comfort index for livestock. International Journal of Biometeorology, v.55, p.455-459, 2011.

SAS - Statistical Analysis System, SAS Companion for the Microsoft Windows Environment. Version 8, Cary: 2007. CD-Rom
Silva, E. C. L.; Modesto, E. C.; Azevedo, M.; Ferreira, M. A.; Dubeux Júnior, J. C. B.; Schuler, A. R. P. Efeitos da disponibilidade de sombra sobre o desempenho, atividades comportamentais e parâmetros fisiológicos de vacas da raça Pitangueiras. Acta Scientiarum. Animal Sciences, v.31, p.295-302, 2009.

Silva, I. M.; Pandorfi, H.; Almeida, G. L. P.; Guiselini, C.; Henrique, H. M. Benefits of automated acclimatization during the pre-milking phase of lactating girolando cows. Engenharia Agrícola, v.31, p.847-856, 2011.

Silva, I. M.; Pandorfi, H.; Vasconcelos, A. J. S.; Laurenti, R.; Guiselini, G. Low cost driver device for microclimate maintenance in the pre-milking of dairy cattle. Engenharia Agrícola, v.33, p.1-10, 2013.

Thom, E. C. The discomfort index. Weatherwise, v.12, p.57-59, 1959.

Vianello, R. L.; Alves. A. R. Climatologia. In: Meteorologia básica e aplicada. Viçosa: UFV, 2006. p.377-444. 